\title{
Connections of geometric measure of entanglement of pure symmetric states to quantum state estimation
}

\author{
Lin Chen, ${ }^{1}$ Huangjun Zhu, ${ }^{1,2}$ and Tzu-Chieh $\mathrm{Wei}^{3}$ \\ ${ }^{1}$ Centre for Quantum Technologies, National University of Singapore, 3 Science Drive 2, Singapore 11754 ${ }^{*}$ \\ ${ }^{2}$ NUS Graduate School for Integrative Sciences and Engineering, Singapore 117597, Singapor $\mathbf{E}^{\dagger}$ \\ ${ }^{3}$ Department of Physics and Astronomy, University of British Columbia, \\ Vancouver, British Columbia V6T 1Z1, Canadd
}

(Dated: November 8, 2018)

\begin{abstract}
We study the geometric measure of entanglement (GM) of pure symmetric states related to rankone positive-operator-valued measures (POVMs) and establish a general connection with quantum state estimation theory, especially the maximum likelihood principle. Based on this connection, we provide a method for computing the GM of these states and demonstrate its additivity property under certain conditions. In particular, we prove the additivity of the GM of pure symmetric multiqubit states whose Majorana points under Majorana representation are distributed within a half sphere, including all pure symmetric three-qubit states. We then introduce a family of symmetric states that are generated from mutually unbiased bases (MUBs), and derive an analytical formula for their GM. These states include Dicke states as special cases, which have already been realized in experiments. We also derive the GM of symmetric states generated from symmetric informationally complete POVMs (SIC POVMs) and use it to characterize all inequivalent SIC POVMs in threedimensional Hilbert space that are covariant with respect to the Heisenberg-Weyl group. Finally, we describe an experimental scheme for creating the symmetric multiqubit states studied in this article and a possible scheme for measuring the permanent of the related Gram matrix.

PACS numbers: 03.67.-a, 03.65.Ud, 03.67.Mn, 03.65.Wj
\end{abstract}

\section{INTRODUCTION}

Quantum entanglement is a crucial resource for quantum computation [1, 2] and other information processing tasks, such as quantum teleportation [3], superdense coding [4] and quantum key distribution [5]. In the past few decades, there have been tremendous efforts in understanding various aspects of entanglement in both bipartite and multipartite settings. One of the central issues in entanglement theory is the characterization and quantification of multipartite entanglement [6, 7]. Among the many approaches to the investigation of entanglement, several geometrically motivated measures have been proposed and proved to be useful, such as relative entropy of entanglement 8, 9], geometric measure of entanglement (GM) [10 12] and logarithmic global robustness 13, 14]. Remarkably, these three measures turn out to be related [15 20].

Among the three measures, GM seems to be the easiest to handle and has thus started to attract attention in recent years. Moreover, its applications have gone beyond entanglement theory. GM is closely related to the construction of optimal entanglement witnesses [11], experimental estimation of entanglement [21], and discrimination of quantum states under local operations and classical communications (LOCC) [15, 16, 22]. Recently, GM

*Electronic address: cqtcl@nus.edu.sg (Corresponding ${ }^{*}$ Author)

$\dagger$ Electronic address: zhuhuangjun@nus.edu.sg

${ }_{\ddagger}$ Electronic address: twei@phas.ubc.ca has also been utilized to determine the universality of resource states for one-way quantum computation [23, 24], and to study generic multipartite pure states as computational resources [20, 25]. In the context of condensed matter physics, GM has been demonstrated to be useful for studying quantum many-body systems, such as characterizing ground state properties and detecting phase transitions [26 32].

Given the above applications of GM, it is thus interesting to explore its connection with other important research fields, such as quantum state estimation 33, 34]. Quantum state estimation is a procedure of inferring the state of a quantum system by general measurementspositive-operator-valued measures (POVMs). It is a central issue of quantum mechanics and a cornerstone of various quantum-information processing tasks, such as quantum computation, quantum communication and quantum cryptography. In this context, many measurement and reconstruction methods have been proposed to estimate the target state. Among the reconstruction methods, an efficient one is the maximum likelihood method, which has been widely used in experiments $33-$ 40.

Concerning measurement schemes, mutually unbiased bases (MUBs) [41 45] and symmetric informationally complete (SIC) POVMs [46 53], which stand for very efficient von Neumann measurements and POVMs [54], respectively, are two focuses in the current research community. Great efforts have been directed to solving their existence problem and understanding their structure [41[44, 46 -48, 50 52]; since they are closely related to the physics in finite-dimensional Hilbert spaces [45, 49, 53]. 
In this article, we aim to establish a general connection between GM and quantum state estimation theory, and MUBs, SIC POVMs in particular. The cross fertilizing of these geometric ideas may bring insights to both research fields. We begin by studying the GM of pure symmetric states related to rank-one POVMs, and establishing a general connection with quantum state estimation theory, especially the maximum likelihood principle [33 37]. Based on this connection, we provide a method for computing the GM of these states and demonstrate its additivity property under certain conditions. In particular, we prove the additivity of the GM of pure symmetric multiqubit states whose Majorana points under Majorana representation [55 59] are distributed within a half sphere, including all pure symmetric three-qubit states. We then introduce a family of symmetric states that are generated from MUBs, and derive an analytical formula for their GM. These states reduce to Dicke states in special cases, which are useful for quantum communication and have been realized in experiments [60 62]. Next, we compute the GM of symmetric states generated from SIC POVMs. This result is then used to characterize all inequivalent SIC POVMs in three-dimensional Hilbert space that are covariant with respect to the HeisenbergWeyl group 46 48, 52]. Finally, we propose an experimental scheme for creating the symmetric multiqubit states studied in this article.

The rest of the article is organized as follows. In Sec. II we establish a general connection between the GM of pure symmetric states and the maximum likelihood principle in quantum state estimation theory. In Sec. III, we prove the additivity of the GM of pure symmetric multiqubit states whose Majorana points are distributed within a half sphere, including all pure symmetric three-qubit states. In Sec. IV, we introduce a family of symmetric states generated from MUBs, and derive an analytical formula for their GM. In Sec. V] we derive the GM of symmetric states generated from SIC POVMs and use it to characterize inequivalent SIC POVMs in three-dimensional Hilbert space. We discuss experimental methods for realizing the symmetrized states of this article in Sec. VI. We conclude with a summary.

\section{GM OF PURE SYMMETRIC STATES: A CONNECTION WITH QUANTUM STATE ESTIMATION}

In this section, we study the GM of pure symmetric states related to rank-one POVMs, and establish a general connection with quantum state estimation theory, especially the maximum likelihood principle. Based on this connection, the GM of many pure symmetric states can be computed analytically, and its additivity property be demonstrated. In the next section, this connection will be used to show the additivity of the GM of pure symmetric multiqubit states whose Majorana points are distributed within a half sphere, including all pure sym- metric three-qubit states. Additional examples related to MUBs and SIC POVMs will be presented in the following sections to illustrate this general idea.

\section{A. Preliminary}

The geometric measure of entanglement measures the maximum overlap between a given state and the set of separable states, or equivalently, the set of pure product states, and is defined as [11, 15]

$$
\begin{aligned}
\Lambda^{2}(\rho) & :=\max _{\sigma \in \mathrm{SEP}} \operatorname{tr}(\rho \sigma)=\max _{|\Phi\rangle \in \mathrm{PRO}}\langle\Phi|\rho| \Phi\rangle, \\
G(\rho) & :=-2 \log \Lambda(\rho) .
\end{aligned}
$$

Here "SEP" denotes the set of separable states, and "PRO" the set of pure product states that fully factorize; "log" has base 2 throughout this article. Any pure product state maximizing Eq. (1) is a closest product state of $\rho$.

For symmetric states, the computation of GM can be greatly simplified due to a result in Refs. [20, 63 65]:

Proposition 1 The closest product state to any pure or mixed symmetric $N$-partite state $\rho$ can be chosen to be symmetric; it is necessarily symmetric if $N \geq 3$ :

$$
\begin{aligned}
\Lambda^{2}(\rho) & =\max _{\left|\varphi_{1}\right\rangle, \ldots,\left|\varphi_{N}\right\rangle}\left(\bigotimes_{j=1}^{N}\left\langle\varphi_{j}\right|\right) \rho\left(\bigotimes_{j=1}^{N}\left|\varphi_{j}\right\rangle\right) \\
& =\max _{|\varphi\rangle}\left\langle\left.\varphi\right|^{\otimes N} \rho \mid \varphi\right\rangle^{\otimes N} .
\end{aligned}
$$

Recently, the GM of pure symmetric three-qubit states have been derived based on this observation [66], and a class of maximally entangled three-qubit states has also been obtained 66, 67]. The problem is still open for more general situations, although progress has been made with respect to the connection between the singular values of a hypermatrix and the GM [68]. Below, we shall provide many examples where analytical solutions can be obtained.

Another key ingredient in our investigation is the maximum likelihood (ML) principle of quantum state estimation [33 37]. Consider state estimation using a rank-one POVM composed of $M$ outcomes that are represented by subnormalized pure projectors $\Pi_{j}=\left|\psi_{j}\right\rangle\left\langle\psi_{j}\right|$ such that $\sum_{j} \Pi_{j}=I$. If we are given $N$ copies of an unknown input state and perform $N$ measurements independently, then the outcome statistics obey a multinomial distribution. Suppose outcome $j$ occurs $n_{j}$ times for $j=1,2 \ldots, M$ $\left(\sum_{j} n_{j}=N\right)$; then the frequency of obtaining outcome $j$ is $f_{j}=n_{j} / N$. In the standard state reconstruction, the estimator is obtained by solving the following set of equations:

$$
\left\langle\psi_{j}|\rho| \psi_{j}\right\rangle=f_{j} \quad \forall j
$$

However, such a solution does not always exist. The ML principle consists in choosing a state $\rho_{\mathrm{ML}}$ that maximizes 
the likelihood functional $\mathcal{L}(\rho)$ as an estimator of the true state 33 37],

$$
\mathcal{L}(\rho)=\prod_{j=1}^{M} p_{j}^{n_{j}}
$$

where $p_{j}=\left\langle\psi_{j}|\rho| \psi_{j}\right\rangle$ is the probability of obtaining outcome $j$ given the input state $\rho$. If there exists a state $\rho_{\mathrm{s}}$ that satisfies Eq. (4), that is, the probabilities $p_{j}$ derived from this state coincide with the frequencies $f_{j}$, then $\rho_{\mathrm{S}}$ is also the maximum point of the likelihood functional. In general, there is an efficient iterative algorithm for finding the ML estimator if the POVM is informationally complete (IC) 33 37]. A POVM is IC if we can reconstruct any input state according to the measurement statistics. If the POVM is not IC, the maximum of the likelihood functional can still be computed efficiently, but the ML estimators are generally not unique. In addition, the ML principle is also applicable when the $\left(\left|\psi_{j}\right\rangle\left\langle\psi_{j}\right|\right)$ 's form an incomplete POVM, that is $\sum_{j}\left|\psi_{j}\right\rangle\left\langle\psi_{j}\right|=\Pi \leq I$ [33 37] (be sure to distinguish "complete" and "informationally complete").

\section{B. Connection}

We are now ready to show the connection between the GM of pure symmetric states and quantum state estimation theory. Following the above notation, assume $\sum_{j}\left|\psi_{j}\right\rangle\left\langle\psi_{j}\right|=\Pi \leq I$, and the largest eigenvalue of $\Pi$ is 1. Define $\left.\Psi\left(\left\{n_{j}\right\}\right)\right\rangle$ as the symmetrized state of the product state $\bigotimes_{j=1}^{M}\left|\psi_{j}\right\rangle^{\otimes n_{j}}$,

$$
\left|\Psi\left(\left\{n_{j}\right\}\right)\right\rangle=c P_{\text {sym }} \bigotimes_{j=1}^{M}\left|\psi_{j}\right\rangle^{\otimes n_{j}},
$$

where $P_{\text {sym }}$ is the projector onto the symmetric subspace, and $c$ is a normalization constant, which can be assumed to be positive without loss of generality. The effect of the projector $P_{\text {sym }}$ is determined by its action on pure product states. The action of $P_{\text {sym }}$ on the tensor product of $N$ single-particle kets $\left|a_{k}\right\rangle$ is given by

$$
P_{\text {sym }} \bigotimes_{k=1}^{N}\left|a_{k}\right\rangle=\frac{1}{N !} \sum_{\sigma \in S_{N}} \bigotimes_{k=1}^{N}\left|a_{\sigma(k)}\right\rangle,
$$

where $S_{N}$ is the symmetry group of $N$ letters. Now suppose $\left|a_{k}\right\rangle$ is the $k$-th member of the multiset consisting of $n_{1}$ copies of $\left|\psi_{1}\right\rangle, n_{2}$ copies of $\left|\psi_{2}\right\rangle$ and so on. Define $A$ as the Gram matrix of the kets $\left|a_{k}\right\rangle$, i.e.,

$$
A_{j k}=\left\langle a_{j} \mid a_{k}\right\rangle, \quad j, k=1,2, \ldots, N
$$

The dependence of $A$ on $\left|\psi_{j}\right\rangle$ and $n_{j}$ has been suppressed to simplify the notation. The normalization constant $c$ is thus given by $c=\sqrt{N ! / \operatorname{perm}(A)}[69$ ], where $\operatorname{perm}(A)$ denotes the permanent of the matrix $A$ (for other connections to permanent, see Ref. 65]).

According to Proposition 1 the GM of $\left|\Psi\left(\left\{n_{j}\right\}\right)\right\rangle$ reads

$$
\begin{aligned}
\Lambda^{2}\left(\left|\Psi\left(\left\{n_{j}\right\}\right)\right\rangle\right) & =\max _{|\varphi\rangle}\left|\left\langle\left.\varphi\right|^{\otimes N} \mid \Psi\left(\left\{n_{j}\right\}\right)\right\rangle\right|^{2} \\
& =\frac{N !}{\operatorname{perm}(A)} \max _{|\varphi\rangle} \prod_{j=1}^{M}\left|\left\langle\varphi \mid \psi_{j}\right\rangle\right|^{2 n_{j}} \\
& \leq \frac{N !}{\operatorname{perm}(A)} \max _{\rho} \mathcal{L}(\rho) \\
& \leq \frac{N !}{\operatorname{perm}(A)} 0 \leq p_{j} \leq 1, \sum_{j} p_{j}=1 \prod_{j=1}^{M} p_{j}^{n_{j}} \\
& =\frac{N !}{\operatorname{perm}(A)} \prod_{j=1}^{M} f_{j}^{n_{j}} .
\end{aligned}
$$

Note that, apart from a constant factor, the functional to be maximized in the second line of the above equation is exactly the likelihood functional $\mathcal{L}(\rho)$ associated with the POVM $\left\{\Pi_{j}\right\}$ if we take $f_{j}$ as "frequency" [see Eq. (5)], except that $\rho$ is restricted to pure states here; this is the reason for the first inequality in Eq. (9). The "frequencies" $f_{j}$ are called compatible if there exists a normalized pure state $\left|\varphi_{\mathrm{s}}\right\rangle$ such that $\left|\left\langle\varphi_{\mathrm{s}} \mid \psi_{j}\right\rangle\right|^{2}=f_{j} \forall j$; that is, the $f_{j}$ 's can coincide with the probabilities derived from a pure state. Note that such a pure state is necessarily contained in the eigenspace to the largest eigenvalue of $\Pi$. The maximum in the last line of Eq. (9) can be obtained if and only if the $f_{j}$ 's are compatible. In that case, the likelihood functional $\mathcal{L}(\rho)$ is maximized at the pure state $\left|\varphi_{\mathrm{s}}\right\rangle$, and $\left|\varphi_{\mathrm{s}}\right\rangle \otimes N$ is a closest product state to $\left|\Psi\left(\left\{n_{j}\right\}\right)\right\rangle$.

Moreover, if the maximum of $\mathcal{L}(\rho)$ can be obtained at a pure state, which is true if the $f_{j}$ 's are compatible, then the GM of $\left|\Psi\left(\left\{n_{j}\right\}\right)\right\rangle$ and any pure symmetric $N$ partite state $|\Phi\rangle$ is additive; that is, $G\left(\left|\Psi\left(\left\{n_{j}\right\}\right)\right\rangle \otimes|\Phi\rangle\right)=$ $G\left(\left|\Psi\left(\left\{n_{j}\right\}\right)\right\rangle\right)+G(|\Phi\rangle)$ (see Ref. [20] for a detailed discussion on the additivity property of GM). We prove this statement in Appendix A. In that case, the GM of $\left|\Psi\left(\left\{n_{j}\right\}\right)\right\rangle$ is equal to its asymptotic regularized quantity and gives a lower bound for asymptotic relative entropy of entanglement and asymptotic logarithmic global robustness [20]. For convenience, we summarize the above observations as the following theorem.

Theorem 2 Suppose there are $M$ kets $\left|\psi_{j}\right\rangle$ (not necessarily normalized) that satisfy $\sum_{j=1}^{M}\left|\psi_{j}\right\rangle\left\langle\psi_{j}\right|=\Pi$, and the largest eigenvalue of $\Pi$ is $1 ; n_{1}, n_{2}, \ldots, n_{M}$ are nonnegative integers with sum $N=\sum_{j} n_{j}$ and $f_{j}=n_{j} / N$. Define $A$ as the Gram matrix of the multiset consisting of $n_{1}$ copies of $\left|\psi_{1}\right\rangle, n_{2}$ copies of $\left|\psi_{2}\right\rangle$ and so on; and $\left|\Psi\left(\left\{n_{j}\right\}\right)\right\rangle=\sqrt{N ! / \operatorname{perm}(A)} P_{\mathrm{sym}} \bigotimes_{j=1}^{M}\left|\psi_{j}\right\rangle^{\otimes n_{j}}$. Then the GM of $\left|\Psi\left(\left\{n_{j}\right\}\right)\right\rangle$ is lower bounded by

$$
G\left(\left|\Psi\left(\left\{n_{j}\right\}\right)\right\rangle\right) \geq-\log \left(\frac{N !}{\operatorname{perm}(A)} \prod_{j=1}^{M} f_{j}^{n_{j}}\right)
$$


the bound is saturated if and only if the $f_{j}$ 's are compatible, that is, there exists a normalized pure state $\left|\varphi_{\mathrm{s}}\right\rangle$ such that

$$
\left|\left\langle\varphi_{\mathrm{s}} \mid \psi_{j}\right\rangle\right|^{2}=f_{j} \quad \forall j .
$$

Moreover, if the maximum of $\mathcal{L}(\rho)$ [see Eq. (5) and (9)] can be obtained at a pure state, which is true if the $f_{j}$ 's are compatible, then the $G M$ of $\left|\Psi\left(\left\{n_{j}\right\}\right)\right\rangle$ and any pure symmetric $N$-partite state $|\Phi\rangle$ is additive; that is,

$$
G\left(\left|\Psi\left(\left\{n_{j}\right\}\right)\right\rangle \otimes|\Phi\rangle\right)=G\left(\left|\Psi\left(\left\{n_{j}\right\}\right)\right\rangle\right)+G(|\Phi\rangle) .
$$

No matter whether Eq. (11) can be satisfied or not, the maximum of the likelihood functional $\mathcal{L}(\rho)$ in Eq. (9) can be computed efficiently with an iterative algorithm 33 37]. It gives an upper bound for $\Lambda^{2}\left(\left|\Psi\left(\left\{n_{j}\right\}\right)\right\rangle\right)$ and thus a lower bound for $G\left(\left|\Psi\left(\left\{n_{j}\right\}\right)\right\rangle\right)$. On the other hand, when Eq. (11) is not satisfied, it is still possible that the maximum of the likelihood functional is obtainable at a pure state. Then the $N$-fold tensor product of the pure state is a closest product state to $\left|\Psi\left(\left\{n_{j}\right\}\right)\right\rangle$, and the GM of $\left|\Psi\left(\left\{n_{j}\right\}\right)\right\rangle$ and any pure symmetric $N$-partite state is additive (see Sec. III for examples). In other words, the compatibility condition on the $f_{j}$ 's is sufficient but not necessary for Eq. (12) to hold. It is really remarkable that we can derive the GM of these symmetric states and its additivity property from the property of the likelihood functional.

Besides, the condition on the largest eigenvalue of $\Pi$ in Theorem 2 is not essential; it is adopted mainly for a closer connection with state estimation theory. If instead the largest eigenvalue of $\Pi$ is $g>0$, then Theorem 2 is still applicable as long as $f_{j}$ is replaced by $g f_{j}$ in Eqs. (10) and (11). One advantage of this alternative convention is that $\left|\psi_{j}\right\rangle$ can now be chosen to be normalized. A simple example in the case of qubits is as follows. Suppose there are $M$ normalized kets $\left|\psi_{j}\right\rangle$ whose Bloch vectors are distributed on a circular cone around the $z$ axis, such that $\sum_{j=1}^{M}\left|\psi_{j}\right\rangle\left\langle\psi_{j}\right|=M\left(I+r \sigma_{z}\right) / 2$ with $0<r<1$; and $|\Psi\rangle=\sqrt{M ! / \operatorname{perm}(A)} P_{\text {sym }} \bigotimes_{j=1}^{M}\left|\psi_{j}\right\rangle$. Then $|\Psi\rangle$ has a unique closest product state $|0\rangle^{\otimes M}$ and its GM is

$$
G(|\Psi\rangle)=-\log \left[\frac{M !}{\operatorname{perm}(A)}\left(\frac{1+r}{2}\right)^{M}\right] .
$$

\section{ADDITIVITY OF GM OF PURE SYMMETRIC THREE-QUBIT STATES}

Every pure symmetric $N$-qubit state $|\Psi\rangle$ can be written in the form in Eq. (6), that is,

$$
|\Psi\rangle \propto P_{\mathrm{sym}} \bigotimes_{j=1}^{M}\left|\psi_{j}\right\rangle^{\otimes n_{j}}
$$

and this representation is unique up to permutations of the $\left|\psi_{j}\right\rangle$ 's and some phase factors. This is well-known as the Majorana representation 55]. Under this representation, each pure symmetric $N$-qubit state corresponds to $N$ points on the Bloch sphere. Following Refs. [58, [59], the $\left|\psi_{j}\right\rangle$ 's are called Majorana points of $|\Psi\rangle$. Recently, Majorana representation has found many applications in the study of multipartite entanglement, such as classification of entanglement of pure symmetric multiqubit states under stochastic LOCC (SLOCC) [56], investigation of the GM of these states and maximally entangled states among them [57 59].

As an important application of the theory developed in the previous section and the Majorana representation, in this section, we prove the additivity of the GM of pure symmetric multiqubit states whose Majorana points are distributed within a half sphere, which is true for all pure symmetric three-qubit states. As we shall see shortly, the structure of the state space and its boundary plays a crucial role in proving this additivity property.

Theorem 3 Suppose $|\Psi\rangle$ is a pure symmetric $N$-qubit state whose Majorana points are distributed within a half sphere under the Majorana representation; then the GM of $|\Psi\rangle$ and any pure symmetric $N$-partite state is additive.

According to Theorem 2, to prove this theorem, it suffices to show that the maximum of the following functional can be obtained at a pure state,

$$
\mathcal{L}(\rho)=\prod_{j=1}^{M}\left(\left\langle\psi_{j}|\rho| \psi_{j}\right\rangle\right)^{n_{j}} .
$$

Without loss of generality, we can assume that the Majorana points of $|\Psi\rangle$ lie within a half sphere with $z \geq 0$, and the Bloch vector of $\rho$ is $\boldsymbol{r}=(x, y, z)$ with $x^{2}+y^{2}+z^{2} \leq 1$. Then it is straightforward to verify that $\mathcal{L}(\rho)$ is nondecreasing with $z$, and thus its maximum can be obtained at the boundary of the Bloch sphere, that is, at a pure state. This completes the proof of Theorem 3 ,

For any pure symmetric three-qubit state, the three Majorana points lie within some half sphere; hence, Theorem 3 is applicable. The same is true for any pure symmetric multiqubit state that has at most three distinct Majorana points.

Corollary 4 The GM of any pure symmetric $N$-qubit state that has at most three distinct Majorana points and any pure symmetric $N$-partite state is additive. In particular, the GM of any pure symmetric three-qubit state and any pure symmetric tripartite state is additive.

Corollary 5 Suppose $|\Psi\rangle$ is a pure symmetric $N$-partite state which can be written in the form

$$
|\Psi\rangle \propto P_{\mathrm{sym}} \bigotimes_{j=1}^{3}\left|\psi_{j}\right\rangle^{\otimes n_{j}}
$$

where $n_{1}+n_{2}+n_{3}=N$; then the GM of $|\Psi\rangle$ and any pure symmetric $N$-partite state is additive. 
Without loss of generality, we can assume the three states $\left|\psi_{j}\right\rangle$ belong to a three-dimensional Hilbert space. As in the case of qubit, they can be seen as three extremal points of the eight-dimensional state space whose origin is the completely mixed state; however, the boundary of the state space is no longer a sphere, and the states on the boundary are not necessarily pure. In addition, we can find a suitable hyperplane passing through the origin such that the three points are on the same side of the hyperplane (or on the hyperplane). According to a similar reasoning that leads to Theorem 3 and Corollary 4 , the maximum of the following functional

$$
\mathcal{L}(\rho)=\prod_{j=1}^{3}\left(\left\langle\psi_{j}|\rho| \psi_{j}\right\rangle\right)^{n_{j}}
$$

can be obtained at a state $\rho_{\mathrm{ML}}$ on the boundary of the state space, whose rank is at most two. If $\rho_{\mathrm{ML}}$ is pure, then we are done. Otherwise, when $\rho$ is restricted to the support of $\rho_{\mathrm{ML}}$, we have

$$
\mathcal{L}(\rho)=\prod_{j=1}^{3}\left(\left\langle\psi_{j}^{\prime}|\rho| \psi_{j}^{\prime}\right\rangle\right)^{n_{j}}
$$

where $\left|\psi_{j}^{\prime}\right\rangle$ is the projection of $\left|\psi_{j}\right\rangle$ onto the support of $\rho_{\mathrm{ML}}$. Now applying the same reasoning that leads to Corollary 4 shows that the maximum of $\mathcal{L}(\rho)$ can be obtained at a pure state. Therefore, the Corollary follows from Theorem 2

Theorem 3 and Corollaries 4 and 5 provide a method for computing the asymptotic GM, which in turn provides a lower bound for the asymptotic relative entropy of entanglement and the asymptotic logarithmic global robustness [20]. They are also useful in the study of multipartite pure states as computational resources, since GM and its additivity property are closely related to whether these states are universal for quantum computation [20, 23 25]. Corollary 4 also implies the multiplicativity of the output purity of the quantum channels associated with pure symmetric three-qubit states according to the Werner-Holevo recipe [20, 70].

\section{SYMMETRIC STATES GENERATED FROM MUTUALLY UNBIASED BASES}

To illustrate the general idea presented in Sec. II in this section we consider the situation where the POVM can be decomposed into a set of von Neumann measurements, in particular the scenario where the bases of the von Neumann measurements are mutually unbiased [45]. We first consider pure symmetric states generated from two bases of the qubit Hilbert space, which reduce to Dicke states as special cases. We then generalize the idea to higher-dimensional Hilbert spaces and point out the role played by mutually unbiasedness.

\section{A. Generalization of Dicke states}

Given $\boldsymbol{r}_{0}=(0,0,1)$ and $\boldsymbol{r}_{1}=(\sin \theta, 0, \cos \theta)$, let $|0\rangle$, $|1\rangle$ denote the eigenbasis of $\boldsymbol{r}_{0} \cdot \boldsymbol{\sigma}=\sigma_{z}$, and $\left|\theta_{+}\right\rangle=$ $\cos (\theta / 2)|0\rangle+\sin (\theta / 2)|1\rangle,\left|\theta_{-}\right\rangle=\sin (\theta / 2)|0\rangle-\cos (\theta / 2)|1\rangle$ the eigenbasis of $\boldsymbol{r}_{1} \cdot \boldsymbol{\sigma}$. Given four nonnegative integers $\left\{n_{j k}\right\}=\left\{n_{00}, n_{01} ; n_{10}, n_{11}\right\}$, let $N=n_{00}+n_{01}+n_{10}+$ $n_{11}, N_{j}=n_{j 0}+n_{j 1}, f_{j k}=n_{j k} / N_{j}$ (assuming $N_{j} \neq 0$ ). Define $A$ as the Gram matrix of the multiset consisting of $n_{00}, n_{01}, n_{10}, n_{11}$ copies of $|0\rangle,|1\rangle,\left|\theta_{+}\right\rangle,\left|\theta_{-}\right\rangle$, respectively; and

$$
\begin{aligned}
\left|\Psi\left(\theta,\left\{n_{j k}\right\}\right)\right\rangle:= & \sqrt{\frac{N !}{\operatorname{perm}(A)}} P_{\text {sym }}\left(|0\rangle^{\otimes n_{00}} \otimes|1\rangle^{\otimes n_{01}}\right. \\
& \left.\otimes\left|\theta_{+}\right\rangle^{\otimes n_{10}} \otimes\left|\theta_{-}\right\rangle^{\otimes n_{11}}\right)
\end{aligned}
$$

Here the permanent perm $(A)$ can be computed efficiently, see Appendix B. Note that $\left|\Psi\left(\theta,\left\{n_{j k}\right\}\right)\right\rangle$ can be seen as a generalization of the Dicke state; it reduces to the Dicke state when $\theta=0, \pi$ or $N_{0}=0$ or $N_{1}=0$. The GM of the Dicke state has been derived in Ref. [11], and its additivity property has been demonstrated in Ref. [20]. When $N_{0}, N_{1} \neq 0$, we have the following theorem.

Theorem 6 The GM of $\left|\Psi\left(\theta,\left\{n_{j k}\right\}\right)\right\rangle$ is lower bounded by

$$
G\left(\left|\Psi\left(\theta,\left\{n_{j k}\right\}\right)\right\rangle\right) \geq-\log \left(\frac{N !}{\operatorname{perm}(A)} f_{00}^{n_{00}} f_{01}^{n_{01}} f_{10}^{n_{10}} f_{11}^{n_{11}}\right)
$$

the bound is saturated if and only if there exists a qubit state $|\varphi\rangle$ such that

$$
\begin{gathered}
|\langle\varphi \mid 0\rangle|^{2}=f_{00}, \quad|\langle\varphi \mid 1\rangle|^{2}=f_{01} \\
\left|\left\langle\varphi \mid \theta_{+}\right\rangle\right|^{2}=f_{10}, \quad\left|\left\langle\varphi \mid \theta_{-}\right\rangle\right|^{2}=f_{11} .
\end{gathered}
$$

When $\theta \neq 0, \pi$, this condition is equivalent to the following one:

$$
\left|h_{0} s_{0}+h_{1} s_{1}\right| \leq 1
$$

where $h_{j}=f_{j 0}-f_{j 1}$, and $\boldsymbol{s}_{0}=(-\cot \theta, 0,1), \boldsymbol{s}_{1}=$ $(\csc \theta, 0,0)$ is the dual basis of $\boldsymbol{r}_{0}, \boldsymbol{r}_{1}$ in the $x-z$ plane.

The $G M$ of $\left|\Psi\left(\theta,\left\{n_{j k}\right\}\right)\right\rangle$ and any pure symmetric $N$ partite state is additive, irrespective whether the condition Eq. 21) is satisfied or not.

We can briefly show Theorem [6 as follows. First Eqs. (20) and (21) can be derived according to a similar reasoning that leads to Theorem 2, Equation (22) has a nice geometric interpretation under the Bloch sphere representation. Suppose the Bloch vector of $|\varphi\rangle$ is $\boldsymbol{s}$. The first two equations in Eq. (21) restrict $s$ to a plane satisfying $\boldsymbol{s} \cdot \boldsymbol{r}_{0}=h_{0}$, which is perpendicular to $\boldsymbol{r}_{0}$, and the last two restrict $\boldsymbol{s}$ to a plane satisfying $\boldsymbol{s} \cdot \boldsymbol{r}_{1}=h_{1}$, which is perpendicular to $\boldsymbol{r}_{1}$. There exists a pure state satisfying Eq. (21) if and only if the intersection of the two planes 
passes through the Bloch sphere; that is, the intersection of the two planes and the $x-z$ plane lies within the unit circle centered at the origin on the $x-z$ plane. This is exactly what Eq. (22) means. In the special case $\theta=\frac{\pi}{2}, \frac{3 \pi}{2}$, where the two bases $|0\rangle,|1\rangle$ and $\left|\theta_{ \pm}\right\rangle=| \pm\rangle$are mutually unbiased, Eq. (22) simplifies to $h_{0}^{2}+h_{1}^{2} \leq 1$. The additivity property of the GM of $\left|\Psi\left(\theta,\left\{n_{j k}\right\}\right)\right\rangle$ follows from Theorem 3. This completes the proof of Theorem 6.

When $n_{01}=n_{00}$ and $n_{11}=n_{10}$, Eq. (21) is trivial to satisfy; and the $N$-fold tensor product of the two eigenstates of $\sigma_{y}$, respectively, are exactly two closest product states to $\left|\Psi\left(\theta,\left\{n_{j k}\right\}\right)\right\rangle$. Besides, Eq. (20) reduces to

$$
G\left(\left|\Psi\left(\theta,\left\{n_{j k}\right\}\right)\right\rangle\right)=-\log \left(\frac{N !}{2^{N} \operatorname{perm}(A)}\right) .
$$

Remarkably, the GM is completely determined by the number of qubits $N$ and perm $(A)$. An interesting example is the state $|\Psi(\theta,\{1,1 ; 1,1\})\rangle$ obtained when $n_{00}=$ $n_{01}=n_{10}=n_{11}=1$. It is a balanced four-qubit Dicke state when $\theta=0, \pi$, and is equivalent to four-qubit GHZ state under a suitable local unitary transformation when $\theta=\frac{\pi}{2}, \frac{3 \pi}{2}$, according to Refs. [56 59, 71]. Calculation shows that $\operatorname{perm}(A)=[7+\cos (2 \theta)] / 2$, hence,

$$
G(|\Psi(\theta,\{1,1 ; 1,1\})\rangle)=\log \frac{7+\cos (2 \theta)}{3} .
$$

The maximum is obtained at $\theta=0, \pi$, where the two bases coincide, and the minimum at $\theta=\frac{\pi}{2}, \frac{3 \pi}{2}$, where the two bases are mutually unbiased.

\section{B. A connection with MUBs}

To generalize the above idea to higher dimension, let $\left|e_{k}^{j}\right\rangle$ for $j=0,1, \ldots, b-1, k=0,1, \ldots, d-1$ be $b d$ singleparticle states such that the $d$ states for given $j$ form an orthonormal basis [45]. Let $n_{j k}$ be a $b \times d$ matrix composed of nonnegative integers, $N=\sum_{j, k} n_{j k}, N_{j}=$ $\sum_{k} n_{j k}$, and $f_{j k}=n_{j k} / N_{j}$ (assuming $N_{j} \neq 0$ ). Define

$$
\left|\Psi\left(\left\{n_{j k}\right\}\right)\right\rangle:=\sqrt{\frac{N !}{\operatorname{perm}(A)}} P_{\mathrm{sym}} \bigotimes_{j, k}\left|e_{k}^{j}\right\rangle^{\otimes n_{j k}} .
$$

This state reduces to a generalized Dicke state when there is only one basis, that is $b=1$. According to the same reasoning as before, the GM of $\left|\Psi\left(\left\{n_{j k}\right\}\right)\right\rangle$ is lower bounded by

$$
G\left(\left|\Psi\left(\left\{n_{j k}\right\}\right)\right\rangle\right) \geq-\log \left(\frac{N !}{\operatorname{perm}(A)} \prod_{j, k} f_{j k}^{n_{j k}}\right)
$$

the bound is saturated if and only if there exists a pure state $|\varphi\rangle$ such that

$$
\left|\left\langle\varphi \mid e_{k}^{j}\right\rangle\right|^{2}=f_{j k} \quad \forall j, k .
$$

In that case, the GM of $\left|\Psi\left(\left\{n_{j k}\right\}\right)\right\rangle$ and any pure symmetric $N$-partite state is additive .
In general, it is not easy to determine whether such a state exists or not; the structure of the state space plays a crucial role here. Here we are content to point out a connection with MUBs. Suppose the $b$ bases $\left|e_{k}^{j}\right\rangle$ are mutually unbiased, and $n_{j k}=n_{j}, f_{j k}=1 / d \forall j, k$. Then satisfying the set of constraints in Eq. (25) amounts to the existence of a pure state that is mutually unbiased with all states $\left|e_{k}^{j}\right\rangle$.

Theorem 7 Suppose $\left|e_{k}^{j}\right\rangle$ for $j=0,1, \ldots, b-1(b \leq d+1)$ and $k=0,1, \ldots, d-1$ are $b$ MUBs, i.e., $\left|\left\langle e_{k}^{j} \mid e_{m}^{l}\right\rangle\right|^{2}=$ $\frac{1}{d}\left(1-\delta_{j, l}\right)+\delta_{j, l} \delta_{k, m}$. Then the $N$-partite $(N=$ $\left.d \sum_{j=1}^{b} n_{j}, n_{j} \geq 1\right)$ symmetric state $\left|\Psi\left(d,\left\{n_{j}\right\}\right)\right\rangle:=$ $\left.\sqrt{N ! / \operatorname{perm}(A)} P_{\text {sym }}\left(\bigotimes_{j=1}^{b} \bigotimes_{k=1}^{d}\left|e_{k}^{j}\right\rangle\right\rangle^{\otimes n_{j}}\right)$ has GM lower bounded by

$$
G\left(\left|\Psi\left(d,\left\{n_{j}\right\}\right)\right\rangle\right) \geq-\log \frac{N !}{d^{N} \operatorname{perm}(A)} ;
$$

the bound is saturated if and only if there exists a pure state that is mutually unbiased to all states $\left|e_{k}^{j}\right\rangle$. If such a state exists, then the GM of $\left|\Psi\left(d,\left\{n_{j}\right\}\right)\right\rangle$ and any pure symmetric $N$-partite state $|\Phi\rangle$ is additive; that is,

$$
G\left(\left|\Psi\left(d,\left\{n_{j}\right\}\right)\right\rangle \otimes|\Phi\rangle\right)=G\left(\left|\Psi\left(d,\left\{n_{j}\right\}\right)\right\rangle\right)+G(|\Phi\rangle) .
$$

For example, the inequality in Eqs. (26) is saturated and (27) is applicable when $b=2$ and the $\left|e_{k}^{0,1}\right\rangle$ 's are the eigenbases of $Z$ and $X$, respectively, where $Z$ and $X$ are phase operator and cyclic shift operator, respectively. They are defined according to their action on the computational basis $\left|e_{k}\right\rangle=\left|e_{k}^{0}\right\rangle$,

$$
\begin{aligned}
Z\left|e_{k}\right\rangle & =\omega^{k}\left|e_{k}\right\rangle, \quad \omega=\mathrm{e}^{2 \pi \mathrm{i} / d} \\
X\left|e_{k}\right\rangle & =\left|e_{(k+1) \bmod d}\right\rangle,
\end{aligned}
$$

where we use "mod" to denote the modulo operation. Since the eigenbases of $X, Z$ and $X Z$ are mutually unbiased [45], the $N$-fold tensor product of any eigenstate of $X Z$ is a closest product state to $\left|\Psi\left(d,\left\{n_{j}\right\}\right)\right\rangle$.

If $d$ is a prime power, there exists a complete set of $d+1$ MUBs [42, 45]. The inequality in Eqs. (26) is saturated and (27) is applicable if $\left|e_{k}^{j}\right\rangle$ 's are chosen from $b$ bases with $1 \leq b \leq d$ from the complete set. However, this is not the case if $b=d+1$, since there is no pure state that is mutually unbiased to all states in a complete set of MUBs 42.

\section{SYMMETRIC STATES GENERATED FROM SIC POVMS}

In a $d$-dimensional Hilbert space, a SIC POVM $46-$ 48, 51 consists of $d^{2}$ outcomes that are subnormalized projectors onto pure states $\Pi_{j}=\frac{1}{d}\left|\psi_{j}\right\rangle\left\langle\psi_{j}\right|$ for $j=1, \ldots, d^{2}$, such that

$$
\left|\left\langle\psi_{j} \mid \psi_{k}\right\rangle\right|^{2}=\frac{1+d \delta_{j k}}{d+1} .
$$


The condition $\sum_{j} \Pi_{j}=I$ is already implied by the above equation and need not be imposed separately. Most known SIC POVMs are generated from a fiducial state under the action of the Heisenberg-Weyl (HW) group, which is generated by the two operators $X, Z$ defined in Eq. (28). A fiducial state $|\psi\rangle$ of the HW group obeys the following equations,

$$
\left|\left\langle\psi\left|X^{k_{1}} Z^{k_{2}}\right| \psi\right\rangle\right|=\frac{1}{\sqrt{d+1}}
$$

for all $\left(k_{1}, k_{2}\right) \neq(0,0) \bmod d$. If $|\psi\rangle$ is a fiducial state, then the $d^{2}$ states $X^{k_{1}} Z^{k_{2}}|\psi\rangle$ for $k_{1}, k_{2}=0,1, \ldots, d-1$ form a SIC POVM that is covariant with respect to the HW group. The Clifford group is the normalizer of the HW group that consists of unitary operators. Likewise, the extended Clifford group is the larger group that contains also anti-unitary operators. For any operator $U$ in the extended Clifford group, $U|\psi\rangle$ is a fiducial state whenever $|\psi\rangle$ is. Fiducial states and SIC POVMs form disjoint orbits under the action of the extended Clifford group. SIC POVMs on the same orbit of the extended Clifford group are equivalent in the sense that they can be transformed into each other with unitary or antiunitary operations [48, 52].

In this section we study the GM of symmetric states generated from SIC POVMs,

$$
\left|\Psi_{d}^{\mathrm{SIC}}\right\rangle:=\sqrt{\frac{d^{2} !}{\operatorname{perm}(A)}} P_{\mathrm{sym}}\left(\left|\psi_{1}\right\rangle \otimes \cdots \otimes\left|\psi_{d^{2}}\right\rangle\right) .
$$

Since the completely mixed state is the only state that satisfies $\left\langle\psi_{j}|\rho| \psi_{j}\right\rangle=1 / d$ for $j=1,2, \ldots, d^{2}$, the GM of $\left|\Psi_{d}^{\mathrm{SIC}}\right\rangle$ cannot be computed according to Theorem 2 . In Appendix $\mathrm{C}$, we derive the GM of $\left|\Psi_{d}^{\mathrm{SIC}}\right\rangle$ by virtue of the special properties of SIC POVMs; the result is summarized in the following theorem.

Theorem 8 Suppose $d^{2}$ normalized states $\left|\psi_{j}\right\rangle$ for $j=$ $1,2, \ldots, d^{2}$ in a d-dimensional Hilbert space satisfy $\sum_{j=1}^{d^{2}}\left|\psi_{j}\right\rangle\left\langle\psi_{j}\right|=d I$, and $A$ is the Gram matrix of the $\left|\psi_{j}\right\rangle$ 's; define $|\Psi\rangle=\sqrt{d^{2} ! / \operatorname{perm}(A)} P_{\text {sym }}\left(\left|\psi_{1}\right\rangle \otimes \cdots \otimes\right.$ $\left.\left|\psi_{d^{2}}\right\rangle\right)$. Then the following four statements are equivalent.

1. The $d^{2}$ states $\left|\psi_{j}\right\rangle$ form a SIC POVM, that is, they satisfy Eq. (29);

2. Up to global phases, the $d^{2}$ states $\left|\psi_{j}\right\rangle^{\otimes d^{2}}$ for $j=$ $1, \cdots, d^{2}$ are the only closest product states to $|\Psi\rangle$; and

$$
\Lambda^{2}(|\Psi\rangle)=\frac{d^{2} !}{(d+1)^{d^{2}-1} \operatorname{perm}(A)} ;
$$

3. The $d^{2}$ states $\left|\psi_{j}\right\rangle^{\otimes d^{2}}$ for $j=1, \cdots, d^{2}$ satisfy

$$
\left|\left\langle\left.\psi_{j}\right|^{\otimes d^{2}} \mid \Psi\right\rangle\right|^{2}=\frac{d^{2} !}{(d+1)^{d^{2}-1} \operatorname{perm}(A)} ;
$$

4. There exists a SIC POVM consisting of $d^{2}$ states $\left|\varphi_{j}\right\rangle$ for $j=1,2, \ldots, d^{2}$ such that $\left|\varphi_{j}\right\rangle^{\otimes d^{2}}$ satisfies

$$
\left|\left\langle\left.\varphi_{j}\right|^{\otimes d^{2}} \mid \Psi\right\rangle\right|^{2}=\frac{d^{2} !}{(d+1)^{d^{2}-1} \operatorname{perm}(A)} .
$$

In dimension two, there is only one orbit of fiducial states, one of them is given by [46 48

$$
\left|\psi_{2}^{\mathrm{f}}\right\rangle=\sqrt{(3+\sqrt{3}) / 6}\left|e_{0}\right\rangle+e^{\mathrm{i} \pi / 4} \sqrt{(3-\sqrt{3}) / 6}\left|e_{1}\right\rangle \text {. }
$$

Moreover, all SIC POVMs are equivalent to the one thus generated. The four states of each SIC POVM form a regular tetrahedron when represented on the Bloch sphere. Calculation shows that $\operatorname{perm}(A)=\frac{8}{3}$, which, together with Theorem 8, implies that

$$
\Lambda^{2}\left(\left|\Psi_{2}^{\mathrm{SIC}}\right\rangle\right)=\frac{1}{3}, \quad G\left(\left|\Psi_{2}^{\mathrm{SIC}}\right\rangle\right)=\log 3 .
$$

This result coincides with that obtained by Martin et al. [57] and Aulbach et al. [59]. They also showed by numerics that the state generated from the SIC POVM is the maximally entangled pure symmetric four-qubit state with respect to GM.

On the other hand, there is a one-parameter family of fiducial states in dimension three [46 48, 52],

$$
\left|\psi_{3}^{\mathrm{f}}(t)\right\rangle=\frac{1}{\sqrt{2}}\left(\left|e_{1}\right\rangle-\mathrm{e}^{\mathrm{i} t}\left|e_{2}\right\rangle\right)
$$

and there is a one-to-one correspondence between orbits of the extended Clifford group and the parameter $t$ for $t \in\left[0, \frac{\pi}{3}\right]$. There are three kinds of orbits, two exceptional orbits corresponding to the endpoints $t=0$ and $t=\frac{\pi}{3}$, respectively, and infinitely many generic orbits corresponding to $0<t<\frac{\pi}{3}$. In addition, there is a oneto-one correspondence between inequivalent SIC POVMs and the parameter $t$ for $0 \leq t \leq \frac{\pi}{9}$; the SIC POVMs on the three orbits $t, \frac{2 \pi}{9}-t, \frac{2 \pi}{9}+t$, respectively, are equivalent under unitary transformations. All these inequivalent SIC POVMs can be classified in terms of geometric phases associated with fiducial states [52]. Here we provide an alternative characterization in terms of GM.

Let $\left|\Psi_{3}^{\mathrm{SIC}}(t)\right\rangle$ denote the symmetric state related to the SIC POVM generated from the fiducial state $\left|\psi_{3}^{\mathrm{f}}(t)\right\rangle$ under the HW group, $A(t)$ the corresponding Gram matrix. Surprisingly, the permanent of $A(t)$ can be given by a simple formula: $\operatorname{perm}(A(t))=\frac{27}{32}[61-\cos (9 t)]$. According to Theorem 8 and Eq. (2),

$$
G\left(\left|\Psi_{3}^{\mathrm{SIC}}(t)\right\rangle\right)=\log \frac{16[61-\cos (9 t)]}{105} .
$$

Figure 1 shows $G(t):=G\left(\left|\Psi_{3}^{\mathrm{SIC}}(t)\right\rangle\right)$ for $0 \leq t \leq \frac{\pi}{3}$. Up to the equivalence class, each SIC POVM is uniquely specified by $G\left(\left|\Psi_{3}^{\mathrm{SIC}}(t)\right\rangle\right)$. There is only a small difference among the GM of symmetric states generated from different SIC POVMs. The minimum and the maximum of the GM are obtained at the exceptional orbits $t=0$ and $t=\frac{\pi}{3}$, respectively. Note that the SIC POVMs on the orbits $t=\frac{2 \pi}{9}$ and $t=\frac{\pi}{9}$ are equivalent to the ones on the orbits $t=0$ and $t=\frac{\pi}{3}$, respectively [52]. 


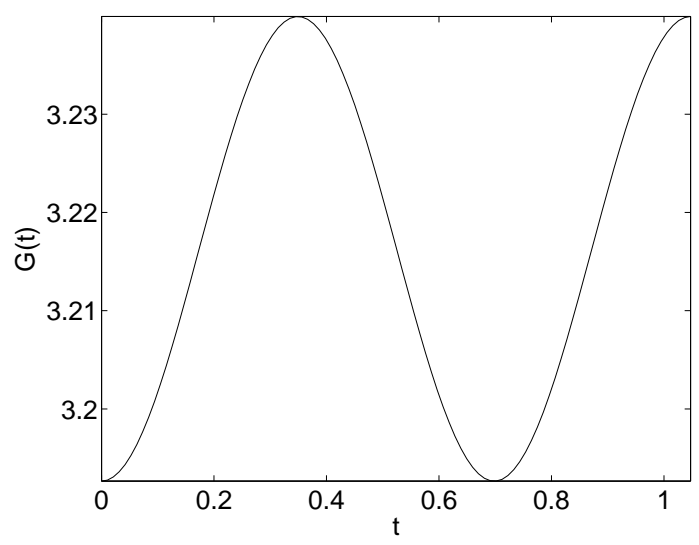

FIG. 1: The GM of symmetric states $\left|\Psi_{3}^{\mathrm{SIC}}(t)\right\rangle$ for $0 \leq$ $t \leq \frac{\pi}{3}$ generated from HW covariant SIC POVMs in threedimensional Hilbert space. Each SIC POVM is uniquely specified by $G(t)$ up to the equivalence class; recall that there is a one-to-one correspondence between inequivalent SIC POVMs and the parameter $t$ for $0 \leq t \leq \frac{\pi}{9}[52$.

\section{CREATING SYMMETRIC MULTI-QUBIT STATES}

In this section, we describe how to create symmetric multi-qubit states mentioned in this article, following the approach by Bastin et al. 71]. For non-qubit systems, a generalization could be made, but we do not know whether suitable physical systems exist.

Imagine we have an atom or trapped ion initially in the excited state $|e\rangle$, and it has two stable ground states, labeled by $|0\rangle$ and $|1\rangle$, which are connected to the excited state via emitting right-circularly $(R)$ and left-circularly $(L)$ polarized light, respectively. If the detection of the emitted photon is $R(L)$ then we know the atom is now in the state $|0\rangle(|1\rangle)$. The photon polarization carries the which-way information. If we place a polarizer in $(|R\rangle+|L\rangle) / \sqrt{2}$ to erase the which-way information and if the detector behind the polarizer clicks, the atom is then projected to an equal superposition of $|0\rangle$ and $|1\rangle$. In general, if the polarizer is in $\alpha|R\rangle+\beta|L\rangle$, after detecting the photon, the atom is in the state $\alpha|0\rangle+\beta|1\rangle$.

Now suppose we have $N$ excited atoms and $N$ photon detectors. A simultaneous detection of $N$ photons will project the $N$-atom state, depending on the polarizers' settings. Suppose the $k$-th polarizer is in $\alpha_{k}|R\rangle+\beta_{k}|L\rangle$. If the $k$-th polarizer and detector sit close to the $k$-th atom and detect the emitted photon by the nearby atom, and if each detector-atom system is far away from each other, then after detection of a photon at every detector, the $N$-atom state is $\otimes_{k}\left(\alpha_{k}|0\rangle_{k}+\beta_{k}|1\rangle_{k}\right)$. Bastin et al. 71] proposed the setup such that the $N$ detectors are placed in the far-field, roughly at an equal distance to all atoms. Because of multipath quantum interference (which-path information is erased), after the detection, the atoms should be in a state that is invariant under permutations, namely,

$$
|\Psi\rangle \propto P_{\mathrm{sym}} \bigotimes_{k}\left(\alpha_{k}|0\rangle_{k}+\beta_{k}|1\rangle_{k}\right)
$$

Thus, using the scheme by Bastin et al. 71], the symmetric multi-qubit states discussed in this article can be realized in principle, not just mathematical objects.

For non-qubit systems, one needs to first identify a physical system that contains an excited state coupled to $d$ longlived sublevels and that the decay into each level is associated with a distinguishable which-way information. Deleting the which-way information from the decay and the which-atom information will enable the creation of symmetric multiqudit states considered in this article. But we have not yet identified such a system.

Next, we discuss a possible measurement of the permanent of $A_{i j}=\left\langle\psi_{i} \mid \psi_{j}\right\rangle$. Suppose one can prepare the following symmetric $N$-particle state

$$
|\Psi\rangle=c P_{\mathrm{sym}} \bigotimes_{j=1}^{N}\left|\psi_{j}\right\rangle,
$$

with the normalization $c=\sqrt{N ! / \operatorname{perm}(A)}$. If one chooses a von Neumann measurement at each particle in the basis consisting of $|\varphi\rangle$ and the remaining orthonormal states, the simultaneous detection of outcome $|\varphi\rangle$ at all sites has the probability

$$
\lambda(\varphi)^{2}=\frac{N !}{\operatorname{perm}(A)} \prod_{j=1}^{N}\left|\left\langle\varphi \mid \psi_{j}\right\rangle\right|^{2} .
$$

Since all $\left|\left\langle\varphi \mid \psi_{j}\right\rangle\right|$ 's are known and $\lambda(\varphi)^{2}$ is obtained from the measurement statistics, $\operatorname{perm}(A)$ can be inferred. Ideally, one prefers $\lambda(\varphi)^{2}$ to be as large as possible, as it represents the probability of obtaining the desired outcome. One can also vary $|\varphi\rangle$; the probability $\lambda(\varphi)^{2}$ is maximized when $|\varphi\rangle^{\otimes N}$ is the closest product state that results in the geometric measure. According to a recent work by Martin et al. [57], the maximal overlap $\Lambda^{2}$ for highly entangled symmetric states decays only inversely proportional to the number of qubits, so the proposed scheme seems to be feasible even for medium size of systems that have been achieved in various physical implementations.

\section{SUMMARY}

We have studied the GM of pure symmetric states related to rank-one POVMs and established its connection with the maximum likelihood principle in quantum state estimation theory. Based on this connection, we provided a method for computing the GM of these states and demonstrated its additivity property under certain conditions. In particular, we proved the additivity of the GM of pure symmetric multiqubit states whose Majorana points are distributed within a half sphere, including all pure symmetric three-qubit states. We then 
introduced a family of symmetric states that are generated from MUBs and derived an analytical formula for their GM. We also derived the GM of symmetric states generated from SIC POVMs and used it to characterize all inequivalent HW covariant SIC POVMs in threedimensional Hilbert space. A scheme for creating the symmetric multiqubit states studied in this article was also proposed. Our studies promise a broad perspective of integrating two important research areas in quantum information science, namely, entanglement characterization and quantum state estimation.

\section{Acknowledgment}

We thank Markus Grassl for discussions on the symmetric states generated from SIC POVMs and for his valuable comments on the manuscript. We also thank Daniel Greenberger for discussions on the connection between the GM of symmetric states and the maximum likelihood principle. H.Z. thanks Berthold-Georg Englert, Yong Siah Teo and Hui Khoon Ng for discussions on the maximum likelihood methods. The Centre for Quantum Technologies is funded by the Singapore Ministry of Education and the National Research Foundation as part of the Research Centres of Excellence programme. T.C.W. acknowledges support from NSERC and MITACS.

\section{Appendix A: Additivity of the GM of $\left|\Psi\left(\left\{n_{j}\right\}\right)\right\rangle$}

In this appendix, we prove Eq. (12) when the maximum of the likelihood functional $\mathcal{L}(\rho)$ can be obtained at a pure state. Equation (12) is equivalent to

$$
\Lambda^{2}\left(\left|\Psi\left(\left\{n_{j}\right\}\right)\right\rangle \otimes|\Phi\rangle\right)=\Lambda^{2}\left(\left|\Psi\left(\left\{n_{j}\right\}\right)\right\rangle\right) \Lambda^{2}(|\Phi\rangle) .
$$

According to the definition, the l.h.s is never smaller than the r.h.s; so it suffices to show that $\Lambda^{2}\left(\left|\Psi\left(\left\{n_{j}\right\}\right)\right\rangle \otimes|\Phi\rangle\right) \leq$ $\Lambda^{2}\left(\left|\Psi\left(\left\{n_{j}\right\}\right)\right\rangle\right) \Lambda^{2}(|\Phi\rangle)$. Suppose $\left|\Psi\left(\left\{n_{j}\right\}\right)\right\rangle$ is shared over the parties $A_{1}, A_{2}, \ldots, A_{N}$, and $|\Phi\rangle$ over the parties $B_{1}, B_{2}, \ldots, B_{N}$; suppose $\left|a_{k}\right\rangle$ is the $k$-th member of the multiset consisting of $n_{1}$ copies of $\left|\psi_{1}\right\rangle, n_{2}$ copies of $\left|\psi_{2}\right\rangle$ and so on. Then according to Proposition 1.

$$
\begin{aligned}
& \Lambda^{2}\left(\left|\Psi\left(\left\{n_{j}\right\}\right)\right\rangle \otimes|\Phi\rangle\right) \\
& =\max _{|\varphi\rangle} \mid\left\langle\left.\varphi\right|^{\otimes N}\left|\left(\left|\Psi\left(\left\{n_{j}\right\}\right)\right\rangle \otimes|\Phi\rangle\right)\right|^{2}\right. \\
& \left.=\frac{N !}{\operatorname{perm}(A)} \max _{|\varphi\rangle}\left|\left(\bigotimes_{k=1}^{N}\left\langle\left.\varphi\right|_{A_{k}, B_{k}} \mid a_{k}\right\rangle_{A_{k}}\right)\right| \Phi\right\rangle\left._{B_{1}, \ldots, B_{N}}\right|^{2} \\
& =\frac{N !}{\operatorname{perm}(A)} \max _{|\varphi\rangle}\left[\left(\bigotimes_{k=1}^{N}\left|\left\langle\left.\varphi\right|_{A_{k}, B_{k}} \mid a_{k}\right\rangle_{A_{k}}\right|^{2}\right)\right. \\
& \left.\left.\times\left|\left(\bigotimes_{k=1}^{N} \frac{\left\langle\left.\varphi\right|_{A_{k}, B_{k}} \mid a_{k}\right\rangle_{A_{k}}}{\left|\left\langle\left.\varphi\right|_{A_{k}, B_{k}} \mid a_{k}\right\rangle_{A_{k}}\right|}\right)\right| \Phi\right\rangle\left._{B_{1}, \ldots, B_{N}}\right|^{2}\right] \\
& \leq \Lambda^{2}(|\Phi\rangle) \frac{N !}{\operatorname{perm}(A)} \max _{\rho} \prod_{k=1}^{N}\left(\left\langle\left. a_{k}\right|_{A_{k}} \rho_{A_{k}} \mid a_{k}\right\rangle_{A_{k}}\right) \\
& =\Lambda^{2}(|\Phi\rangle) \frac{N !}{\operatorname{perm}(A)} \max _{\rho} \mathcal{L}(\rho) \\
& =\Lambda^{2}\left(\left|\Psi\left(\left\{n_{j}\right\}\right)\right\rangle\right) \Lambda^{2}(|\Phi\rangle) \text {. }
\end{aligned}
$$

Here the last equality is due to our assumption that the maximum of the likelihood functional $\mathcal{L}(\rho)$ can be obtained at a pure state.

\section{Appendix B: $\operatorname{perm}(A)$ in Eq. (19)}

Note that the entries of the Gram matrix $A$ in Eq. (19) only take on five different values $0,1, \sin (\theta / 2), \pm \cos (\theta / 2)$. In contrast with the computation of the permanent of a generic matrix, the permanent of $A$ can be computed efficiently as follows:

$$
\begin{aligned}
& \operatorname{perm}(A)=\left(\prod_{j, k=0}^{1} n_{j k} !\right) \sum_{\{a, b, c, f, g\}}(-1)^{g-a}\left(\cos \frac{\theta}{2}\right)^{2 f+2 g} \\
& \times\left(\sin \frac{\theta}{2}\right)^{2 a+2 b+2 c-2 g}\left(\begin{array}{c}
n_{00} \\
a, b, n_{00}-a-b
\end{array}\right) \\
& \times\left(\begin{array}{c}
n_{01} \\
c, f, n_{01}-c-f
\end{array}\right)\left(\begin{array}{c}
n_{10} \\
g, a+c-g, n_{10}-a-c
\end{array}\right) \\
& \times\left(\begin{array}{c}
n_{11} \\
a+b-g, f+g-a, n_{11}-b-f
\end{array}\right),
\end{aligned}
$$

where the summation is restricted to the set of nonnegative integers $\{a, b, c, f, g\}$ satisfying the following constraints,

$$
\begin{aligned}
& a+b \leq n_{00}, \quad c+f \leq n_{01}, \quad a+c \leq n_{10}, \quad b+f \leq n_{11}, \\
& g \leq a+b, \quad g \leq a+c, \quad f+g \geq a .
\end{aligned}
$$




\section{Appendix C: Proof of Theorem 8}

We begin by proving the implication $1 \Rightarrow 2$. According to Proposition 1 and the definition of a SIC POVM,

$$
\begin{aligned}
\Lambda^{2}(|\Psi\rangle) & =\Lambda^{2}\left(\left|\Psi_{d}^{\mathrm{SIC}}\right\rangle\right)=\max _{|\varphi\rangle}\left|\left\langle\left.\varphi\right|^{\otimes d^{2}} \mid \Psi_{d}^{\mathrm{SIC}}\right\rangle\right|^{2} \\
& =\frac{d^{2} !}{\operatorname{perm}(A)} \max _{|\varphi\rangle} \prod_{j=1}^{d^{2}}\left|\left\langle\varphi \mid \psi_{j}\right\rangle\right|^{2}
\end{aligned}
$$

Here the maximization is subjected to the completeness condition $\sum_{j}\left|\left\langle\varphi \mid \psi_{j}\right\rangle\right|^{2}=d$ and the following condition

$$
\sum_{j}\left|\left\langle\varphi \mid \psi_{j}\right\rangle\right|^{4}=\frac{2 d}{d+1}
$$

since a SIC POVM is a 2-design [47]: $\sum_{j}\left|\psi_{j}\right\rangle\left\langle\psi_{j}\right| \otimes$ $\left|\psi_{j}\right\rangle\left\langle\psi_{j}\right|=[2 d /(d+1)] \Pi_{\text {sym }}$, where $\Pi_{\text {sym }}$ is the projector onto the bipartite symmetric subspace. Hence,

$$
\begin{aligned}
\Lambda^{2}\left(\left|\Psi_{d}^{\mathrm{SIC}}\right\rangle\right) & \leq \frac{d^{2} !}{\operatorname{perm}(A)} \max _{\substack{0 \leq p_{j} \leq 1, \sum_{j} p_{j}=d \\
\sum_{j} p_{j}^{2}=\frac{2 d}{d+1}}} \prod_{j=1}^{d^{2}} p_{j} \\
& =\frac{d^{2} !}{(d+1)^{d^{2}-1} \operatorname{perm}(A)}
\end{aligned}
$$

The maximum in the above equation is obtained if all $p_{j}$ 's are equal to $1 /(d+1)$ except one of them, which is equal to 1 . A state can satisfy these conditions if and only it belongs to the SIC POVM, hence, the implication $1 \Rightarrow 2$ follows.

The implication $2 \Rightarrow 3$ is obvious. The implication $3 \Rightarrow 1$ and 4 can be shown as follows,

$$
\begin{aligned}
\left|\left\langle\left.\psi_{j}\right|^{\otimes d^{2}} \mid \Psi\right\rangle\right|^{2} & =\frac{d^{2} !}{\operatorname{perm}(A)} \prod_{k=1}^{d^{2}}\left|\left\langle\psi_{j} \mid \psi_{k}\right\rangle\right|^{2} \\
& \leq \frac{d^{2} !}{\operatorname{perm}(A)}\left(\frac{\sum_{k=1, k \neq j}^{d^{2}}\left|\left\langle\psi_{j} \mid \psi_{k}\right\rangle\right|^{2}}{d^{2}-1}\right)^{d^{2}-1} \\
& =\frac{d^{2} !}{(d+1)^{d^{2}-1} \operatorname{perm}(A)}
\end{aligned}
$$

The inequality is saturated if and only if $\left|\left\langle\psi_{j} \mid \psi_{k}\right\rangle\right|^{2}=$ $1 /(d+1), \forall j, k$ and $j \neq k$; that is, the $\left|\psi_{j}\right\rangle$ 's form a SIC POVM, which implies 1 and 4 .

It remains to show the implication $4 \Rightarrow 1$

$$
\begin{aligned}
\left|\left\langle\left.\varphi_{j}\right|^{\otimes d^{2}} \Psi\right\rangle\right|^{2} & =\frac{d^{2} !}{\operatorname{perm}(A)} \prod_{k=1}^{d^{2}}\left|\left\langle\varphi_{j} \mid \psi_{k}\right\rangle\right|^{2} \\
& =\frac{d^{2} !}{\operatorname{perm}(A)} \prod_{k=1}^{d^{2}}\left(\prod_{j=1}^{d^{2}}\left|\left\langle\varphi_{j} \mid \psi_{k}\right\rangle\right|^{2}\right)^{\frac{1}{d^{2}}} \\
& \leq \frac{d^{2} !}{\operatorname{perm}(A)} \max _{|\psi\rangle} \prod_{j=1}^{d^{2}}\left|\left\langle\varphi_{j} \mid \psi\right\rangle\right|^{2} \\
& \leq \frac{d^{2} !}{(d+1)^{d^{2}-1} \operatorname{perm}(A)}
\end{aligned}
$$

Here the second inequality follows from Eq. (48), recall that the $\left|\varphi_{j}\right\rangle$ 's form a SIC POVM. The inequalities are saturated if and only if the $\left|\psi_{j}\right\rangle$ 's are the same as the $\left|\varphi_{j}\right\rangle$ 's up to some permutation and phase factors; which implies 1.
[1] R. Jozsa, e-print arXiv:quant-ph/9707034 (1997).

[2] R. Raussendorf and H. J. Briegel, Phys. Rev. Lett. 86, 5188 (2001).

[3] C. H. Bennett, G. Brassard, C. Crépeau, R. Jozsa, A. Peres, and W. K. Wootters, Phys. Rev. Lett. 70, 1895 (1993).

[4] C. H. Bennett and S. J. Wiesner, Phys. Rev. Lett. 69, 2881 (1992).

[5] A. Ekert, Phys. Rev. Lett. 67, 661 (1991).

[6] M. B. Plenio and S. Virmani, Quant. Inf. Comp. 7, 1 (2007).

[7] R. Horodecki, P. Horodecki, M. Horodecki, and K. Horodecki, Rev. Mod. Phys. 81, 865 (2009).

[8] V. Vedral, M. B. Plenio, M. A. Rippin, and P. L. Knight, Phys. Rev. Lett. 78, 2275 (1997).

[9] V. Vedral and M. B. Plenio, Phys. Rev. A 57, 1619 (1998).

[10] A. Shimony, Ann. N. Y. Acad. Sci. 755, 675 (1995).

[11] T.-C. Wei and P. M. Goldbart, Phys. Rev. A 68, 042307 (2003).

[12] D. C. Brody and L. P. Hughston, J. Geom. and Phys.
38, 19 (2001).

[13] G. Vidal and R. Tarrach, Phys. Rev. A 59, 141 (1999).

[14] A. W. Harrow and M. A. Nielsen, Phys. Rev. A 68, 012308 (2003).

[15] M. Hayashi, D. Markham, M. Murao, M. Owari, and S. Virmani, Phys. Rev. Lett. 96, 040501 (2006).

[16] M. Hayashi, D. Markham, M. Murao, M. Owari, and S. Virmani, Phys. Rev. A 77, 012104 (2008).

[17] T.-C. Wei, M. Ericsson, P. M. Goldbart, and W. J. Munro, Quantum Inf. Comput. 4, 252 (2004).

[18] D. Cavalcanti, Phys. Rev. A 73, 044302 (2006).

[19] T.-C. Wei, Phys. Rev. A 78, 012327 (2008).

[20] H. Zhu, L. Chen, and M. Hayashi, New J. Phys. 12, 083002 (2010).

[21] O. Gühne, M. Reimpell, and R. F. Werner, Phys. Rev. Lett. 98, 110502 (2007).

[22] D. Markham, A. Miyake and S. Virmani, New J. Phys. 9, 194 (2007).

[23] M. Van den Nest, W. Dür, A. Miyake, and H. J. Briegel, New J. Phys. 9, 204 (2007).

[24] C. E. Mora, M. Piani, A. Miyake, M. Van den Nest, W. 
Dür, and H. J. Briegel, Phys. Rev. A 81, 042315 (2010).

[25] D. Gross, S. T. Flammia, and J. Eisert, Phys. Rev. Lett. 102, 190501 (2009).

[26] T.-C. Wei, D. Das, S. Mukhopadyay, S. Vishveshwara, and P. M. Goldbart, Phys. Rev. A 71, 060305 (R) (2005).

[27] R. Orús, Phys. Rev. Lett. 100, 130502 (2008).

[28] R. Orús, S. Dusuel, and J. Vidal, Phys. Rev. Lett. 101, 025701 (2008).

[29] R. Orús, Phys. Rev. A 78, 062332 (2008).

[30] T.-C. Wei, Phys. Rev. A 81, 062313 (2010).

[31] R. Orús, and T.-C. Wei, arXiv:0910.2488, to appear in Phys. Rev. B.

[32] W. Son, L. Amico, S. Pascazio, R. Fazio, and V. Vedral, arXiv:1001.2656

[33] Quantum State Estimation, edited by M. G. A. Paris, J. Řeháček, Lecture Notes in Physics, Vol. 649 (Springer, Berlin, 2004).

[34] A. I. Lvovsky and M. G. Raymer, Rev. Mod. Phys. 81, 299 (2009).

[35] Z. Hradil, Phys. Rev. A 55, R1561 (1997).

[36] J. Řeháček, Z. Hradil and M. Ježek, Phys. Rev. A 63, 040303(R) (2001).

[37] J. Řeháček, Z. Hradil, Phys. Rev. A 75, 042108 (2007).

[38] D. F. V. James, P. G. Kwiat, W. J. Munro, and A. G. White, Phys. Rev. A 64, 052312 (2001).

[39] M. D. de Burgh, N. K. Langford, A. C. Doherty, and A. Gilchrist, Phys. Rev. A 78, 052122 (2008).

[40] R. B. A. Adamson and A. M. Steinberg, Phys. Rev. Lett. 105, 030406 (2010).

[41] I. D. Ivanović, J. Phys. A: Math. Gen. 14, 3241 (1981).

[42] W. K. Wootters and B. D. Fields, Annals of Physics 191, 363 (1989).

[43] W. K. Wootters, arXiv:quant-ph/0406032 (2004).

[44] D. M. Appleby, AIP Conf. Proc. 1101, 223 (2009).

[45] T. Durt, B.-G. Englert, I. Bengtsson and K. Życzkowski, International Journal of Quantum Information 8, 535 (2010).

[46] G. Zauner, Ph.D. thesis, University of Vienna, 1999; available online at http://www.mat.univie.ac.at/ neum /papers/physpapers.html

[47] J. M. Renes, R. Blume-Kohout, A. J. Scott, and C. M. Caves, J. Math. Phys. 45, 2171 (2004).

[48] D. M. Appleby, J. Math. Phys. 46, 052107 (2005).

[49] D. M. Appleby, H. B. Dang, and C. A. Fuchs,
arXiv:0707.2071 [quant-ph].

[50] D. M. Appleby, S. T. Flammia, and C. A. Fuchs, arXiv:1001.0004 [quant-ph].

[51] A. J. Scott, and M. Grassl, J. Math. Phys. 51, 042203 (2010).

[52] H. Zhu, J. Phys. A: Math. Theor. 43, 305305 (2010).

[53] C. A. Fuchs, arXiv:quant-ph/0205039.

[54] A. J. Scott, J. Phys. A: Math. Gen. 39, 13507 (2006).

[55] E. Majorana, Nuovo Cimento 9, 43 (1932).

[56] T. Bastin, S. Krins, P. Mathonet, M. Godefroid, L. Lamata, and E. Solano, Phys. Rev. Lett. 103, 070503 (2009).

[57] J. Martin, O. Giraud, P. A. Braun, D. Braun, T. Bastin, Phys. Rev. A 81, 062347 (2010).

[58] D. Markham, arXiv:1001.0343 [quant-ph].

[59] M. Aulbach, D. Markham, M. Murao, New J. Phys. 12, 073025 (2010).

[60] N. Kiesel, C. Schmid, G. Tóth, E. Solano, and H. Weinfurter, Phys. Rev. Lett. 98, 063604 (2007).

[61] W. Wieczorek, R. Krischek, N. Kiesel, P. Michelberger, G. Tóth, and H. Weinfurter, Phys. Rev. Lett. 103, 020504 (2009).

[62] R. Prevedel, G. Cronenberg, M. S. Tame, M. Paternostro, P. Walther, M. S. Kim, and A. Zeilinger, Phys. Rev. Lett. 103, 020503 (2009).

[63] R. Hübener, M. Kleinmann, T.-C. Wei, C. G. Guillén, and O. Gühne, Phys. Rev. A 80, 032324 (2009).

[64] M. Hayashi, D. Markham, M. Murao, M. Owari, and S. Virmani, J. Math. Phys. 50, 122104 (2009).

[65] T.-C. Wei and S. Severini, J. Math. Phys. 51, 092203 (2010).

[66] L. Chen, A. Xu and H. Zhu, Phys. Rev. A 82, 032301 (2010).

[67] S. Tamaryan, T.-C. Wei, and D. Park, Phys. Rev. A 80, 052315 (2009).

[68] J. J. Hilling and A. Sudbery, J. Math. Phys. 51, 072102 (2010).

[69] T.-C. Wei, Phys. Rev. A 81, 054102 (2010).

[70] R. F. Werner and A. S. Holevo, arXiv:quant-ph/0203003.

[71] T. Bastin, C. Thiel, J. von Zanthier, L. Lamata, E. Solano, and G. S. Agarwal, Phys. Rev. Lett. 102, 053601 (2009). 